\title{
Vaginal Myomectomy for Semipedunculated Cervical Myoma during Pregnancy
}

\author{
Mikitaka Obara, MD ${ }^{1} \quad$ Yuko Hatakeyama, MD ${ }^{1} \quad$ Yasushi Shimizu, MD ${ }^{1}$ \\ ${ }^{1}$ Department of Obstetrics \& Gynecology, HIRAKA General Hospital, \\ Yokote City, Akita, Japan \\ Am J Perinatol Rep 2014;4:37-40.

\begin{abstract}
Address for correspondence Dr. Mikitaka Obara, MD, Department of Obstetrics \& Gynecology, HIRAKA General Hospital, 3-1 Yatsuguchi, Maego, Yokote city, Akita, Postcode 013-8610, Japan (e-mail: ob6329@gmail.com).
\end{abstract}

Abstract
Keywords
- prolapsed myoma
- semipedunculated
leiomyoma
- vaginal myomectomy
- pregnancy

Objective Cases of prolapsed myoma in which pregnancy was carried to full term or near term after vaginal myomectomy are very rare. Previously, only two such cases have been reported. In addition, both those patients had a pedunculated leiomyoma, which could be treated by twisting or clamping. Here, we report a case of a patient who was able to carry her pregnancy to term despite vaginal myomectomy for semipedunculated myoma at 13 weeks of pregnancy.

Study Design This study is a case presentation.

Results The myoma nucleus was removed by making an incision on the surface of the mass. Systemic and transvaginal antibiotics were prescribed after the surgery. Uterine contractions, short cervix, or cervical funneling was not observed in the remaining duration of pregnancy.

Conclusion While performing myomectomy during pregnancy, removal of the myoma nucleus is safer than twisting. In addition, postoperative administration of local or systemic antibiotic agents does not adversely affect pregnancy.
Prolapsed myoma during pregnancy is uncommon, and few cases of patients undergoing vaginal myomectomy during pregnancy have been reported. ${ }^{1-3}$ Thus far, only in two such cases were the patients able to carry their pregnancy to full term or near term after the surgery, and both had a pedunculated leiomyoma, which could be treated by twisting or clamping of the pedicle. A previous case of a patient with a semipedunculated leiomyoma who had undergone vaginal myomectomy, at 13 weeks of pregnancy caused premature membrane rupture 2 weeks after the surgery. ${ }^{1}$ Here, we report a case of a patient who underwent vaginal myomectomy at 13 weeks of pregnancy for semipedunculated myoma without avulsion and basilar ligature, and the patient was able to continue her pregnancy to term.

\section{Case Report}

A 31-year-old nulligravida presented with cervical myoma, which was first diagnosed 6 months before pregnancy. She visited our hospital for pregnancy evaluation at 6 weeks gestation, at which time a cervical myoma was diagnosed by transvaginal ultrasonography. At 7 weeks, she experienced transient vaginal bleeding and severe lower abdominal pain, with spontaneous improvement of symptoms. Speculum examination at 9 weeks of pregnancy revealed a uterine myoma extending from the cervical canal into the vagina (-Fig. 1). Magnetic resonance imaging (MRI) showed a submucous myoma measuring $6.2 \times 4.3 \mathrm{~cm}$, arising from the posterior wall of the uterine cervix (-Fig. 2). The internal os was closed. Because of intermittent vaginal bleeding from 11 weeks of pregnancy, the patient was considered to be at risk for major bleeding. She was fully informed of the risks of miscarriage or hysterectomy occurring due to vaginal myomectomy. After her consent, surgery was scheduled at 13 weeks and 6 days of pregnancy.

The patient was transferred to our hospital at 13 weeks and 1 day of pregnancy due to severe vaginal bleeding. Arterial bleeding from the surface of the leiomyoma filling the vagina was controlled by pressure. To prevent rebleeding from the several engorged vessels, vaginal myomectomy was received

September 30, 2013 accepted after revision December 23, 2013

published online

March 12, 2014
Dol http://dx.doi.org/

10.1055/s-0034-1370352. ISSN 2157-6998.
Copyright (c) 2014 by Thieme Medical Publishers, Inc., 333 Seventh Avenue, New York, NY 10001, USA. Tel: +1(212) 584-4662.
License terms

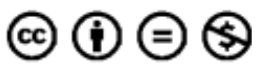




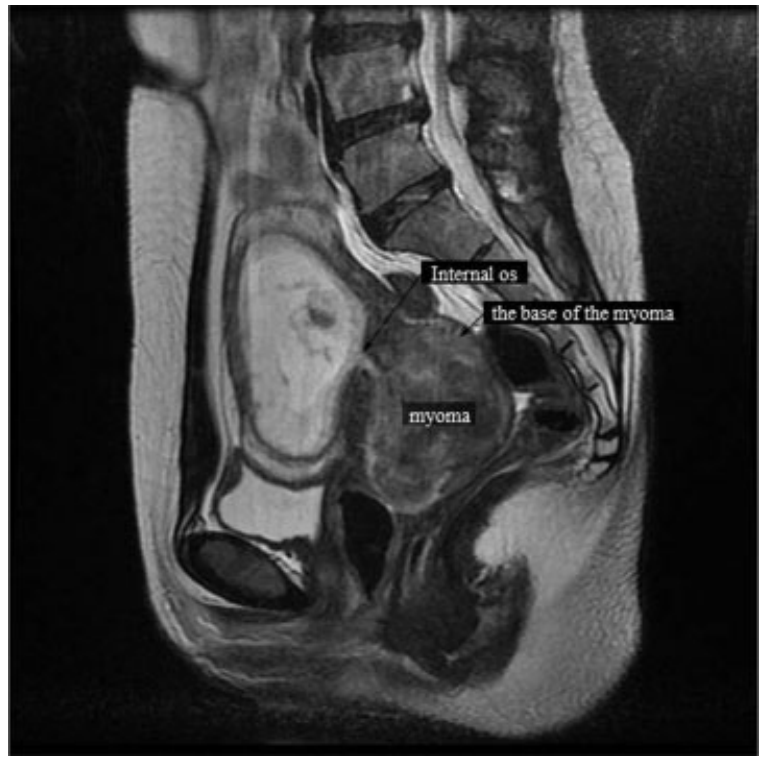

Fig. 1 Ultrasound image of prolapsed submucosal myoma.

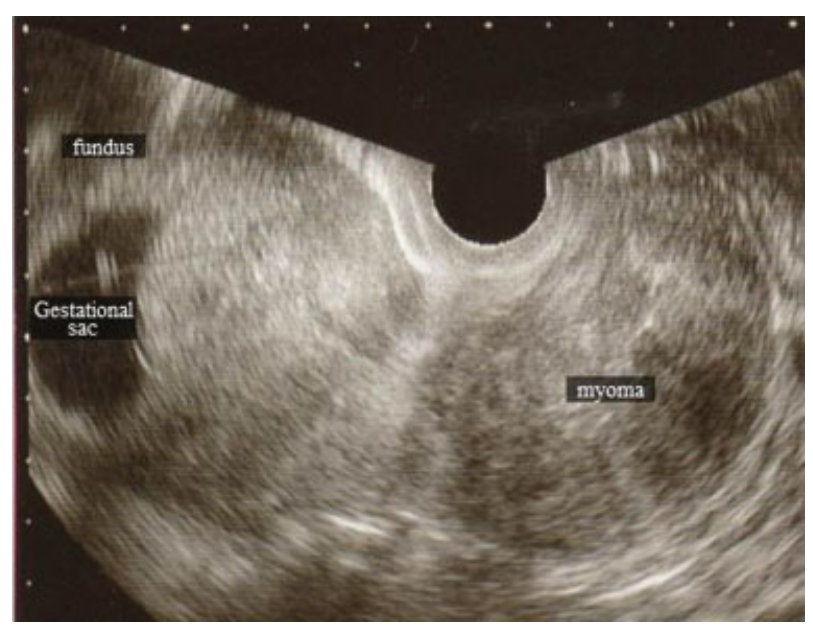

Fig. 2 Magnetic resonance images of prolapsed submucosal myoma.

performed, under spinal anesthesia, on the same day. The myoma was semipedunculated and the entire perimeter of its base was too thick to be palpable, making twisting and clamping of the base impossible. We, therefore, made an incision on the surface of the mass to remove the myoma nucleus carefully. The residual 3 to $4 \mathrm{~cm}$ concavity at the posterior wall of the cervix was sutured with polyglactin 910 , and hemostasis was established. The amount of operative bleeding was $145 \mathrm{~mL}$.

Following surgery, the patient received systemic and transvaginal antibiotics as infection prophylaxis. There was no evidence of uterine contractions, short cervix, or cervical funneling. The patient was discharged at 12 days after the surgery, and her subsequent course of pregnancy was unremarkable.

Because of oligohydramnios, labor was induced with oxytocin after mechanical cervical dilatation using a metreurynter at 40 weeks gestation. The patient gave birth to a male infant weighing $2,755 \mathrm{~g}$ by spontaneous vaginal delivery. The amount of bleeding during delivery was $350 \mathrm{~mL}$. Her postpartum course was uneventful, and she was discharged home with the infant on postpartum day 5 .

\section{Conclusion}

In most cases of vaginal myomectomy during pregnancy, surgery was performed postdelivery. Only four cases of patients undergoing vaginal myomectomy during pregnancy have been reported. ${ }^{4}$ In three of these four cases, surgical treatment consisted of twisting or clamping of the base of a pedunculated leiomyoma. Of these three patients, two patients were able to carry pregnancy to full term or near term, whereas one patient who underwent vaginal myomectomy at 18 weeks had premature membrane rupture 30 days after the surgery. ${ }^{4}$ The fourth patient underwent myomectomy for a cervical semipedunculated myoma with thick base at 13 weeks of pregnancy. Although surgery was successful, she experienced premature membrane rupture 2 weeks postsurgery with subsequent miscarriage. ${ }^{1}$

We consider the following factors significant for surgical treatment and postoperative care for myoma delivery during pregnancy.

Location of the myoma base: Conditions are more favorable for myomectomy when the base of the myoma is located in the cervical canal rather than in the body of the uterus. The cervical canal is commonly dilated by myoma delivery from the external os to the attachment site of myoma. Bleeding is therefore more easily controlled if the myomectomy wound is located in the cervical canal. In our patient, we were able to palpate the myomectomy wound in the cervical canal and suture it to stop bleeding. In contrast, if the base were located in the body of the uterus, direct control of bleeding in the pregnant uterus would have been almost impossible. MRI is technically superior to ultrasonography for identifying the base, and we believe it is an indispensable preoperative examination for vaginal myomectomy.

Technique: In our patient, the base of the myoma was too thick to be palpable, making it difficult to perform avulsion, ligation of the base, and clamp procedures. Although myomectomy did not appear to be feasible in this case, we were able to remove the myoma nucleus with particular attention to the release layer, as done in common myomectomy. As this procedure makes it possible to control each bleeding point, this may be a safer technique than twisting. Vaginal myomectomy has not been recommended in cases where the base of myoma is inaccessible. However, we have shown that when diagnostic imaging identifies the myoma base in the cervical canal, the base may be gradually identified and detached even if it cannot be accessed early in the surgical procedure.

Prevention of spontaneous abortion, preterm delivery, and premature rupture: The importance of preventing spread of infection to the uterus is self-evident. Because the myomectomy wound was located in the uterine cervix, wound inflammation may easily spread to the chorion. 
Postoperative cervicitis may occur because the external os of the uterus is dilated. We suspect that this surgery increases the risk of chorioamnionitis and premature rupture. In the four previously described cases of successful cervical canal submucous myomectomy, two patients developed premature membrane rupture. Our patient received systemic antibiotics, 1 week of chloramphenicol and metronidazole by vaginal suppository, until normal vaginal bacterial flora was restored. This treatment might prevent premature membrane rupture in this case. In our patient, the internal cervical os was closed at the time of surgery, however, and more aggressive pharmacotherapy may be necessary for patients with a dilated internal os.

Our experience suggests that vaginal myomectomy may be successfully performed with adequate preoperative evalua- tion and appropriate technique. In such cases, postoperative infection prophylaxis increases the likelihood that pregnancy can be carried to term.

\section{References}

1 Kilpatrick CC, Adler MT, Chohan L. Vaginal myomectomy in pregnancy: a report of two cases. South Med J 2010;103(10): 1058-1060

2 Demirci F, Somunkiran A, Safak AA, Ozdemir I, Demirci E. Vaginal removal of prolapsed pedunculated submucosal myoma during pregnancy. Adv Ther 2007;24(4):903-906

3 Scott RB, Spence JM Jr. Delivering submucous myoma complicating pregnancy. Am J Obstet Gynecol 1951;62(2):447-449

4 Straub HL, Chohan L, Kilpatrick CC. Cervical and prolapsed submucosal leiomyomas complicating pregnancy. Obstet Gynecol Surv 2010;65(9):583-590 